\title{
Melanoma cutáneo asociado a la mutación del gen BRAF en dos hospitales de la ciudad de Guatemala
}

\author{
Cutaneous melanoma associated with the mutation of the BRAF gene in two hospitals from \\ Guatemala City \\ Elisa C. Hernández-López ${ }^{1 *}$, José L. Rodríguez-Peralto² \\ ${ }^{1}$ Centro de Investigaciones Biomédicas, Facultad de Ciencias Médicas, Universidad de San Carlos de Guatemala, Guatemala; \\ ${ }^{2}$ Departamento de Anatomía Patológica, Hospital Universitario 12 de octubre, Universidad Complutense de Madrid, \\ Madrid, España. \\ *Autor al que se dirige la correspondencia: elisaherlo@gmail.com
}

Recibido: 20 de febrero 2019 / Revisión: 16 de enero 2020 / Aceptado: 03 de marzo 2020

\section{Resumen}

L os estudios de melanoma en Guatemala han sido pocos y únicamente se ha evaluado el aspecto clínico e histológico. El objetivo del presente estudio fue determinar la proporción de casos de melanoma cutáneo por morfología, inmunohistoquímica y mutación del gen BRAF en pacientes con diagnóstico de melanoma en dos centros de referencia, Instituto de Cancerología e Instituto de Dermatología en Guatemala. El estudio es de tipo descriptivo, retrospectivo, transversal. El tipo de muestreo es no probabilístico, con una muestra por conveniencia de 100 casos de tejidos de piel de pacientes, caracterizados por edad, sexo y localización del tumor. Los estudios moleculares incluyeron la determinación de la mutación de la proteína BRAF, por la técnica de PCR-RT. Los resultados muestran que el sexo más afectado es el femenino (54\%). El grupo etario con mayor número de casos es entre 56-75 años (44\%). El tipo histológico predominante es el melanoma lentiginoso acral (59 \%) y la localización más frecuente es en miembro inferior (71\%). No se encontraron casos de melanoma lentigo maligno. La mutación del gen BRAF se encontró en el $6 \%$ de los casos, lo que representa un dato importante para el pronóstico y tratamiento del paciente. Por ser uno de los primeros estudios que incluyen el factor molecular, abre paso a una línea de investigación que permita dar continuidad a los pacientes con melanoma en Guatemala, lo que permitirá determinar factores pronóstico y predictivos, así como tratamientos de los casos en estudio.

Palabras claves: Tipo histológico, inmunohistoquímica, gen BRAF

\begin{abstract}
Q tudies of melanoma in Guatemala have been few and only the clinical and histological aspects have been $\checkmark$ evaluated. The objective of this study was to determine the proportion of cases of cutaneous melanoma by morphology, immunohistochemistry and mutation of the BRAF gene in patients diagnosed with Melanoma in two important reference centers, Institute of Cancerology (Incan) and Institute of Dermatology of Guatemala (Inderma). The study is descriptive, retrospective, transversal. The type of sampling is non-probabilistic, with a convenience sample calculation of 100 cases of patient skin biopsies, characterized by age, sex and tumor anatomic location. Molecular studies included the determination of the BRAF protein mutation by means of the RT-PCR technique. Results show that the most affected sex is the female (54\%). Age group with the highest number of cases is between 56 and 75 years old ( $44 \%$ of cases). The histological type that predominated is acral lentiginous melanoma (59\%) and the most frequent location is in the lower limb (71 \%). No cases of malignant lentigo melanoma were found in the cases studied. The BRAF gene mutation was found in $6 \%$ of the cases, which represents an important data for the prognosis and treatment of the patient. In addition, being one of the first studies that include the molecular factor, it opens the way to a line of research that allows patients with melanoma to continue in Guatemala. This would allow to determine prognostic and predictive factors, as well as treatments of the cases under study.
\end{abstract}

Keywords: Histological type, immunohistochemistry, BRAF gene, melanoma

La reproducción total o parcial del contenido e imágenes de esta publicación se rige de acuerdo a normas internacionales sobre protección a los derechos de autor, con criterio especificados en la licencia Creative Commons (CC BY-NC-SA 4.0) 


\section{Introducción}

El melanoma cutáneo, se ha incrementado desde hace aproximadamente 20 años, y se estima que en EE.UU. se diagnostican 70,000 nuevos casos al año y es la causa de muerte de casi 10,000 personas anualmente (Hernández \& Nieweg, 2014). La mayoría de los melanomas se localizan en la piel y menos frecuentemente en mucosas, pero dado el origen de los melanocitos que derivan de la cresta neural, pueden observarse en otras localizaciones como retina y meninges (Herrera \& Flores, 2010). En los últimos años ha existido un crecimiento exponencial de los casos de melanoma, en varios países europeos el aumento de incidencia anual supera el $2 \%$ existiendo diversas disparidades entre países (Forsea, del Marmol, de Vries, \& Geer, 2012).

El riesgo de desarrollar un melanoma en un individuo nacido en la actualidad es del $1.88 \%$, lo que equivale a que una de cada 53 personas desarrollará un melanoma a lo largo de su vida. El 41 \% de los melanomas se diagnostican antes de los 55 años, siendo este un cáncer muy importante en relación a la reducción en la esperanza de vida de los pacientes (Sánchez et al., 2017).

La exposición al sol en la infancia y más de una quemadura de sol abrasador se asocia con un mayor riesgo de melanoma (Whiteman, Whiteman, \& Green, 2011).

Como en cualquier neoplasia, el pronóstico depende de la etapa. En el momento de su presentación, el $85 \%$ de los pacientes tienen una enfermedad localizada; el $13 \%$, una enfermedad regional (metástasis ganglionares), y sólo el $5 \%$, metástasis a distancia. A pesar de ello, la supervivencia varía enormemente entre pacientes en una misma etapa debido a factores pronósticos e histológicos propios del tumor; probablemente el más importante sea el nivel de invasión o nivel de Breslow (Hernández \& Nieweg, 2014).

Los melanomas se dividen clínicamente por sus fases de crecimiento radial o no tumorogénica en la que el tumor se encuentra in situ y su crecimiento es horizontal dentro de la epidermis y dermis papilar, con grupos de 15 células sin presencia de mitosis y las células tumorales carecen de capacidad para metastatizar. Los tumores en fase de crecimiento radial incluyen: melanoma de extensión superficial, melanoma lentigo maligno y melanoma lentiginoso acral. En esta fase del crecimiento tumoral o vertical, los podemos clasificar en melanoma nodular y la última fase que serían los melanomas raros como los localizados en mucosa genital, oral y conjuntival (Calonge, Brenn, Lazar, \& McKee, 2011).

La clasificación molecular de los melanomas muestra que los melanomas sin daño solar crónico tienen frecuentes mutaciones de BRAF, NRAS y pérdida del cromosoma 10, mientras que en los melanomas con daño solar crónico es infrecuente la mutación de BRAF $\mathrm{y}$ frecuentemente se incrementa el número de copias del gen CCND1 (ciclina D1) (Mishra, Ha, Rieker, Sviderskaya, \& Brennett, 2010). La disección del genoma del melanoma revela un número variable de mutaciones. Una importante vía es la de RAS/actividad mitótica de proteincinasa (MAPK), la cual regula la proliferación celular y sobrevivencia de varios tipos celulares (Curtin et al., 2005). Dichos autores dividieron a los melanomas cutáneos y mucosos en cuatro grupos relacionados con distintos patrones de exposición solar y diferente localización anatómica: melanomas sobre piel sin daño solar crónico (o melanoma relacionado con la exposición solar intermitente); melanomas sobre piel con daño solar crónico (que corresponde al melanoma sobre lentigo maligno); melanomas acrales, y melanomas de mucosas. Hallaron que, mientras el $81 \%$ de los melanomas en piel sin daño solar crónico tenía mutaciones de BRAF o de NRAS (que eran mutuamente excluyentes), la mayoría de melanomas de los otros tres grupos, los tres con un patrón histológico lentiginoso, no presentaban mutaciones de estos genes, pero sí amplificaciones de los genes CDK4 y CCND1 (ciclina D1) y/o aberraciones genéticas de c-Kit que incluían mutaciones y amplificaciones (Curtin, Busam, Pinkel, \& Bastian, 2006).

Se estima que el $50 \%$ de los melanomas cutáneos expresan una mutación activa de BRAF. Al estar activada esta oncoproteína, estimula la proliferación celular e inhibir la apoptosis. El medicamento aprobado por la FDA desde el 2011 para el tratamiento de melanoma metastásico, ha sido el Vemurafenib, un inhibidor específico de la señalización intracelular producida por el BRAF mutado. Otro medicamento que tiene indicación para la mutación BRAF V600E, es el Dabrafenib. Es un inhibidor selectivo de BRAF, aprobado por la FDA en el 2013 para pacientes con melanoma metastásico con prueba positiva para mutación de BRAFV600E (Qu et al., 2013; Sosman et al., 2012)

En Guatemala existen algunos estudios que evidencian la prevalencia de melanoma en la población y el cáncer de piel en general, ocupa el tercer lugar en la frecuencia en Guatemala. En los registros observados en el Instituto de Dermatología (Inderma) y en el Insti- 
tuto de Cancerología (Incan) comprendidos entre enero del 2003 hasta diciembre del 2007, de 38,982 primeras consultas 401 correspondieron a cáncer de piel con una prevalencia del melanoma de 0.4 por cada 1,000 (Incan, 2006; Inderma, 2007).

En el presente estudio se realizó un análisis de una serie de casos de melanoma en la población guatemalteca, describiéndose los hallazgos morfológicos, inmunohistoquímicos y moleculares, como la determinación de la mutación del gen BRAF, para utilizarlo en la prevención y manejo terapéutico de estos pacientes, con el objeto de implementar en un futuro cercano técnicas de diagnóstico molecular para el diagnóstico de melanoma en Guatemala.

\section{Materiales y métodos}

Fueron recolectados 100 muestras de bloques de parafina de los archivos del Incan y del Inderma en la ciudad de Guatemala, con previa autorización de los Comités de Investigación y ética de cada Hospital, que correspondía a pacientes con diagnóstico de melanoma y que consultaron en ambas instituciones de salud. La muestra seleccionada fue por conveniencia, determinándose que la muestra seleccionada era representativa. Las muestras se procesaron a partir de bloques de parafina cuyos cortes fueron teñidos con hematoxilina y eosina. Las muestras para estudios de inmunohistoquímica se realizaron en tejidos con diagnóstico sugestivo de melanomas amelanóticos, utilizando el equipo de inmunohistoquímica automatizado Dako 48 Link, a las que se realizaron las pruebas de inmunohistoquímica: Melan A, HMB 45, S100, confirmándose el diagnóstico de melanoma. Se realizaron pruebas de biología molecular por PCR-RT, para la detección de la mutación del gen BRAF, utilizándose el equipo de Cobas ${ }^{\circledR} 4800$ BRAF V600E. Las pruebas moleculares y de inmunohistoquímica, se realizaron con la colaboración del Laboratorio de Anatomía Patológica del Hospital Universitario 12 de Octubre, Madrid, España.

Los niveles de invasión o niveles de Clark (León, Villaverde, González, \& Soto, 2013) fueron utilizados para clasificar los tumores utilizados en el estudio, ya que estos describen la invasión de la tumoración en los diferentes niveles cutáneos. El nivel I describe los tumores puramente epidérmicos; el nivel II indica que la tumoración atraviesa la unión dermoepidérmica con invasión de la dermis papilar; el nivel III describe las tumoraciones que ocupan toda la dermis papilar expandiéndola; el nivel IV indica que la tumoración infiltra la dermis reticular y el nivel V indica la invasión del tejido graso subcutáneo. El pronóstico empeora al aumentar el nivel. Además, fue utilizado el índice de Breslow para determinar el grosor de los tumores estudiados.

\section{Resultados}

El presente estudio comprendió una serie de casos de pacientes con diferentes tipos de melanoma desde el punto de vista clínico, morfológico y molecular. Se logró determinar el tipo histológico de melanoma más frecuente, localización y mutación del Gen BRAF. Las características clínicas y demográficas de los casos a estudio (Tabla 1) evidencian un ligero incremento de los casos en el sexo femenino (54 \%). La edad promedio se encontró en 62 años. La localización anatómica que predominó es en los miembros inferiores (71\%) específicamente en áreas acrales.

Las características histológicas de pacientes con melanoma (Tabla 2) muestran que el melanoma acral representa el $58 \%$ de los casos, observándose principalmente en las extremidades inferiores, seguido por el

Tabla 1

Características clinicas y demográficas de pacientes con melanoma

\begin{tabular}{lll}
\hline Características & \multicolumn{2}{c}{$\mathrm{n}=100$} \\
\hline Genero & Femenino & 54 \\
& Masculino & 46 \\
Edad & Media: & 62 años \\
& Rango & 18 a 89 años \\
Localización del & Tronco & 8 \\
Melanoma & Extremidad & 9 \\
& superior & \\
& $\begin{array}{l}\text { Extremidad } \\
\text { inferior }\end{array}$ & 71 \\
& Otras & 12 \\
& Presente & 49.4 \\
Úlcera & Ausente & 50.5 \\
\hline
\end{tabular}


Tabla 2

Características histológicas de pacientes con melanoma

\begin{tabular}{|c|c|c|}
\hline Variables & $\mathrm{n}=1$ & \\
\hline \multirow[t]{4}{*}{ Tipo histológico } & MES & 8 \\
\hline & MLA & 58 \\
\hline & $\mathrm{MN}$ & 25 \\
\hline & Mucoso & 9 \\
\hline \multirow[t]{2}{*}{ Crecimiento } & Radial & 25 \\
\hline & Vertical & 75 \\
\hline \multirow[t]{2}{*}{ Mitosis } & $\mathrm{Si}$ & 70 \\
\hline & No & 30 \\
\hline \multirow[t]{5}{*}{ Nivel de Clark } & I & 6.6 \\
\hline & II & 9 \\
\hline & III & 34 \\
\hline & IV & 21 \\
\hline & $\mathrm{V}$ & 29.7 \\
\hline \multirow[t]{4}{*}{ Índice de Breslow } & $<1 \mathrm{~mm}$ & 41 \\
\hline & $1.1 \mathrm{a} 2 \mathrm{~mm}$ & 20 \\
\hline & $2.1 \mathrm{a} 4 \mathrm{~mm}$ & 20 \\
\hline & $>4 \mathrm{~mm}$ & 19 \\
\hline \multirow{2}{*}{ Gen BRAF } & Mutado & 6 \\
\hline & No Mutado & 94 \\
\hline
\end{tabular}

melanoma nodular con un $25 \%$. La distribución de casos por nivel de Clark se encuentra predominantemente en el nivel III con un 34.0\% de los casos seguido por el nivel V con un $29.6 \%$. De los casos estudiados se encontró melanomas amelanóticos en el $5 \%$ de los casos, confirmándose por medio de pruebas de inmunohistoquímica, que mostraron positividad a los anticuerpos HMB 45 y Melan A, en el $100 \%$. El anticuerpo S100 mostró positividad en un $75 \%$ de los casos. El índice de Breslow muestra que el $41 \%$ de los tumores media menos de $1 \mathrm{~mm}$. Por último, el gen BRAF se encontró mutado únicamente en el $6 \%$ de los casos observados.

\section{Discusión}

En los últimos años se ha incrementado progresivamente el interés acerca del melanoma, un tipo altamente agresivo y potencialmente letal de cáncer de piel (Christensen et al., 2013). La sobrevivencia a cinco años de los casos con melanomas primarios localizados recientemente diagnosticados (AJCC estadios I y II) es de aproximadamente un $80 \%$, comparada con un $35 \%$ cuando los ganglios linfáticos están comprometidos (AJCC estadio III) (Calonge et al., 2011).

Cuando existen metástasis a distancia la sobrevivencia a cinco años desciende al $10 \%$. En los últimos años ha existido un crecimiento exponencial de los casos melanoma, en varios países europeos. El aumento de incidencia anual supera el $2 \%$. (Acosta, Fierro, Velásquez, \& Rueda, 2009; Alla et al., 2010).

Los datos epidemiológicos obtenidos en este estudio se muestran en la Tabla 1, donde se mostró una media de edad de 62 años como la edad al diagnóstico de esta patología. Además, se observó en un porcentaje mucho mayor en las extremidades inferiores, representando el $71 \%$ de los casos. El melanoma nodular se observó en un $25 \%$ de la población guatemalteca que representa el segundo más frecuente y es similar a los hallazgos descritos en otros países y que representa de un 15 a $30 \%$, observándose principalmente en tronco. Estos datos son muy similares a los obtenidos en otros estudios latinoamericanos que estudian la epidemiología de los melanomas (Alfaro-Sánchez et al., 2016; Iribarren, Sepúlveda, Hidalgo, \& Madariaga, 2005).

BRAF es una serina/treoninakinasa que activa la vía de transducción de la quinasa MAP/ERK. Entre las mutaciones hay activadores de BRAF, la más frecuente es la V600E, resultado de la transversión timina a adenina en el nucleótido 1799 (T1799A). Esta mutación ha sido reportada en 40 a $60 \%$ de los melanomas que ocurren en población caucásica, siendo más frecuente en los inducidos por radiación solar que aparecen en piel previamente sana, que en aquellos que aparecen en piel con daño solar crónico, en mucosas y en zonas acrales (Blessing et al., 1998; Volkovova, Bilanicova1, Bartonova, Letašiová, \& Dusinska, 2012). En la población asiática esta mutación ha sido observada en el $25 \%$ de los pacientes estudiados (Bertram et al., 2004; Bishop et al., 2002). No hemos encontrado reportes sobre la frecuencia de mutación V600E de BRAF en poblaciones mediterráneas ni latinoamericanas.

En el presente estudio se pretendió hacer un análisis de una serie de casos de melanoma de la población 
de Guatemala, localizado en cara, tronco y acrales para determinar la mutación del gen BRAF por medio de técnicas de patología molecular y con el fin último de utilizarlo para el tratamiento y manejo terapéutico de estos pacientes. En la Tabla 3 se muestra el porcentaje de casos que presentaron una mutación en el gen BRAF, siendo únicamente el $6 \%$. Esto representa una cantidad menor al ser comparada con la de otras poblaciones, por lo que es necesario realizar estudios similares en las poblaciones latinoamericanas. Esto permitirá en un futuro implementar de forma rutinaria dichas técnicas de patología molecular en Guatemala.

Por último, la distribución de casos por nivel de Clark se encuentra predominantemente en el nivel III con un $34.0 \%$ de los casos seguido por el nivel $\mathrm{V}$ con un $29.6 \%$. El índice de Breslow muestra que el $41 \%$ de los tumores medía menos de $1 \mathrm{~mm}$. Estos datos pueden variar debido a diferentes factores que no fueron evaluados dentro de este estudio. La supervivencia se ve influenciada fuertemente por la etapa de diagnóstico. Factores de pronóstico importantes en el tumor primario son el grosor del tumor y la ulceración. Tumores en la cabeza, el cuello y el tronco tienen un peor pronóstico, y la tasa de supervivencia específica de la enfermedad disminuye con el incremento de la edad al momento del diagnóstico.

Los resultados en este estudio son una aproximación preliminar a algunas características epidemiológicas del melanoma en Guatemala y su relación con la mutación del gen BRAF, sin embargo, es necesario continuar con los estudios moleculares y epidemiológicos de esta enfermedad. Esto permitirá el desarrollo del campo diagnóstico, así como el seguimiento predictivo y pronósticos de los casos de estudio. Finalmente, es importante resaltar el impacto de los estudios moleculares en la determinación del tratamiento necesario de cada paciente. La presencia de la mutación del Gen BRAF en casos de melanoma no acral, es de vital importancia, para el tratamiento del melanoma especialmente en aquellos pacientes con metástasis. Actualmente se está utilizando las inmunoterapias para el tratamiento de melanoma, sin embargo este tipo de pruebas cobran importancia para el tratamiento farmacológico del paciente.

\section{Agradecimientos}

Los autores desean agradecer el apoyo del Instituto de Cancerología (Incan) y del Instituto de Dermatología (Inderma).

\section{Referencias}

Acosta, A., Fierro, E., Velásquez, V., \& Rueda, X. (2009). Melanoma: Patogénesis, clínica e histopatología. Revista de la Asociación Colombiana de Dermatología, 17(2), 87-108.

Alfaro-Sánchez, A., García-Hidalgo, L., CasadosVergara, R., Rodríguez-Cabral, R., Piña-Osuna, A., \& Sánchez-Ramos, A. (2016). Cáncer de piel. Epidemiología y variedades histológicas, estudio de cinco años en el noreste de México. Dermatología Revista Mexicana, 60(2), 106-113.

Alla, V., Engelmann, D., Niemetz, A., Pahnke, J., Schmidt, A., Kunz, M., ... Pützer, B. (2010). E2F1 in melanoma progression and metastasis. Journal of the National Cancer Institute, 102(2), 127-133. https://doi.org/10.1093/jnci/djp458.

Bertram, C., Gaut, R., Barrett, J., Randerson-Moor, J., Whitaker, L., Turner, F., ... Swerdlow, A. (2004). An assessment of a variant of the DNA repair gene XRCC3 as a possible nevus ormelanoma susceptibility genotype. Journal of Investigative Dermatology, 122(2), 429-432. https://doi. org/10.1046/j.0022-202X.2003.12541.X

Bishop, D., Demenais, F., Goldstein, A., Bergman, W., Bishop, J., Paillerets, D., ... Tucker, M. (2002). Geographical variation in the penetrance of CDKN2A mutations for melanoma. Journal of the National Cancer Institute, 94(12), 894-903. https://doi.org/10.1093/jnci/94.12.894

Blessing, K., Sanders, D., \& Grant, J. (1998). Comparison of immunohistochemical staining of the novel antibody melan-A with $\mathrm{S} 100$ protein and HMB-45 in malignant melanoma and melanoma variants. Histopathology, 32(2), 139-146. https:// doi.org/10.1046/j.1365-2559.1998.00312.x

Calonge, E., Brenn, T., Lazar, A., \& McKee, P. (2011). McKee 's Pathology of Skin. Edinburgh: Elsevier/ Saunders.

Curtin, J., Busam, K., Pinkel, D., \& Bastian, B. (2006). Somatic activation of KIT in distinct subtypes of melanoma. Journal of Clinical Oncology, 24(26), 4340-4346. https://doi. org/10.1200/JCO.2006.06.2984 
Curtin, J., Fridlyand, J., Kageshita, T., Patel, H., Busam, K.., Kutzner, H., ... Bastian, B. (2005). Distinct sets of genetic alterations in melanoma. New England Journal of Medicine, 353(20), 2135 2147. https://doi.org/10.1056/NEJMoa050092

Forsea, A. M., del Marmol, V., de Vries, E., Bailey, E. E., \& Geller, A. C. (2012). Melanoma incidence and mortality in Europe: New estimates persistent disparities. British Journal of Dermatology, 167(5), 1124-1130. https://doi.org/10.1111/ j.1365-2133.2012.11125.x

Hernández, J. F., \& Nieweg, O. (2014). Melanoma cutáneo, diagnóstico y tratamiento actuales. Revista Gaceta Médica de México, 150(2), 175178

Herrera, N., \& Flores, A. (2010). El melanoma en México. Revista de Especialidades MédicoQuirúrgicas, 15(3), 161-164.

Instituto de Cancerología. (2006). Registro del Cáncer del Instituto de Cancerología Registro Hospitalario años 2006-2010. Registro de Mortalidad por Cáncer. Recuperado de https:// www.ligacancerguate.org/

Instituto Nacional de Dermatología. (2007). Registro de cáncer de Piel. Guatemala: Recuperado de http:// www.indermaguatemala.com/

Iribarren, O., Sepúlveda, M., Hidalgo, J., \& Madariaga, J. (2018). Estudio epidemiológico de melanoma maligno en la IV Región de Chile. Cuadernos de Cirugía, 19(1), 33-38. https://doi.org/10.4206/ cuad.cir.2005.v19n1-06

León, M. V., Villaverde, R. M., González, J. L., \& Soto, M. Á. M. (2013). Melanoma. MedicinePrograma de Formación Médica Continuada Acreditado, 11(26), 1597-1607.

Mishra, P. J., Ha, L., Rieker, J., Sviderskaya, E. V., Bennett, D. C., Oberst, M. D., ... Merlino, G. (2010). Dissection of RAS downstream pathways in melanomagenesis: a role for Ral in transformation. Oncogene, 29(16), 2449-2456. https://doi.org/10.1038/onc.2009.521
Qu, K., Pan, Q., Zhang, X., Rodriguez, L., Zhang, K., Li, H., ...Waldman, F. (2013). Detection of BRAF V600 mutations in metastatic melanoma comparison of the Cobas 4800 and Sanger Sequencing Assays. Journal of Molecular Diagnostics,15(6), 790-795. https://doi. org/10.1016/j.jmoldx.2013.07.003.

Sánchez-Tabernero, S., García-Alvarez, C., MuñozMoreno, M. F., Diezhandino, P., Alonso-Martínez, P., de Frutos-Baraja, J. M., ... Saornil, M. A. (2017). Pattern of local recurrence after I-125 episcleral brachytherapy for uveal melanoma in a Spanish referral ocular oncology unit. American Journal of Ophthalmology, 180, 39-45. https:// doi.org/10.1016/j.ajo.2017.05.019

Sosman, J. A., Kim, K. B., Schuchter, L., González, R., Pavlick, A. C., Weber, J. S., \& Ribas, A. (2012). Survival in BRAF V600-mutant advanced melanoma treated with vemurafenib. New England Journal Medicine, 366, 707-714. https://doi.org/10.1056/NEJMoa1112302

Volkovova, K., Bilanicova, D., Bartonova, A., Letašiová, S., \& Dusinska, M. (2012). Associations between environmental factors and incidence of cutaneous melanoma. Review. Environmental Health, 11 Suppl 1(Suppl 1), S12. https://doi. org/10.1186/1476-069X-11-S1-S12

Whiteman, D. C., Whiteman, C. A., \& Green, A. C. (2011). Childhood sun exposure as a risk factor for melanoma: A systematic review of epidemiologic studies. Cancer Causes Control, 12(1), 69-82. https://doi.org/10.1023/a:1008980919928 